\title{
Lesões de Cárie Proximais na Dentição Decídua: Freqüência de Lesões Homólogas
}

\author{
MARIATH, Adriela Azevedo Souza ${ }_{*}^{*}$ \\ BRESSANI, Ana Eliza Lemes \\ PITONI, Carla Moreira* \\ FOSSATI, Ana Cristina* \\ ARAÚJO, Fernando Borba de
}

\begin{abstract}
RESUMO
O objetivo deste estudo transversal observacional foi avaliar a experiência de cárie na superfície proximal de molares decíduos quanto à ocorrência de comportamento bilateral e ao envolvimento de superfícies adjacentes. A população estudada foi o arquivo de pacientes do Ambulatório de Odontopediatria (UFRGS). Das 670 fichas de pacientes avaliadas, 208 possuíam radiografias interpro-ximais bilaterais. As fichas dos indivíduos que apresentaram lesão de cárie e/ou restauração proximal (kappa intra-examinador: 0,84), com adequado contraste e ausência de sobreposição foram selecionados para a análise. As imagens radio-gráficas foram avaliadas aleatoriamente de acordo com a presença de lesões proximais/restaurações e posteriormente foi analisada a freqüência de acometimento em superfícies homólogas e adjacentes. Assim, 64 pares de radiografias interproximais compuseram a amostra, com ocorrência de $81 \%$ de lesões cariosas em faces homólogas. Dentre estas, $58 \%$ apresentaram mais de um par de superfícies homólogas com lesão cariosa. Além disso, $48 \%$ dos indivíduos mostraram exclusivamente lesões de cárie em pares homólogos. As superfícies distais dos primeiros molares inferiores foram as mais acometidas pela ocorrência bilateral (41\%). Outro aspecto analisado foi a ocorrência simultânea de lesões em faces proximais adjacentes: em $59 \%$ das crianças, ambas as faces de contato apresentaram lesão cariosa diagnosticada através da imagem radiográfica.

A partir da metodologia utilizada, concluiu-se que lesões cariosas de molares decíduos apresentam com-portamento bilateral em faces homólogas, o que representa uma informação útil durante a investigação de lesões em pacientes com experiência de cárie.
\end{abstract}

PALAVRAS-CHAVE:

Cárie dentária. Dente decíduo. Lesão cariosa proximal. Radiografia interproximal

\section{REVISÃO DA LITERATURA}

A superfície proximal é um sítio muito relevante na expressão de doença cárie (MEJARE et al., 2004). O diagnóstico e o manejo clínico da lesão cariosa nesta superfície apresentam características peculiares em função da presença de contato anatômico com o dente adjacente (GRONDAHL et al., 1992). Com o intuito de realizar o diagnóstico o mais precocemente possível, torna-se necessário associar métodos capazes de identificar lesões cariosas iniciais. A radiografia interproximal apresenta acurácia elevada para a superfície proximal, inclusive superior a qualquer outra superfície dentária (PITTS, 1984; WENZEL, 1998; UPRICHARD et al., 2000).

Estudos clínicos comparam a acurácia do exame clínico e radiográfico na detecção de cárie proximal. Considerando as lesões cariosas identificadas através da associação do exame clínico e radiográfico, apenas $30 \%$ foram identificadas so- mente pelo exame clínico, enquanto aproximadamente $95 \%$ foram diagnosticadas pelo radiográfico isoladamente (HOPCRAFT;MORGAN, 2005). Dados obtidos com crianças também evidenciam a restrição do diagnóstico clínico isoladamente, visto apenas $60 \%$ das lesões terem sido identificadas pelo mesmo (MACHIULSKIENE et al., 1999). Em confluência com os dados anteriores, uma estudo transversal de crianças de cinco anos de idade (Suécia) constatou que $12 \%$ apresentaram pelo menos uma lesão em dentina e $33 \%$ pelo menos uma lesão em esmalte que somente foi identificada com o exame radiográfico (ANDERSON et al., 2005). Ainda, quanto menor é a prevalência de cárie na população, mais subestima-se seu diagnóstico, uma vez que a acurácia dos meios diaponíveis para a detecção de cárie é menor para as lesões mais incipientes

( VAN

RIJKOM;VERDONSCHOT, 1995). Em uma amostra de adultos jovens, mais de
$60 \%$ dos indivíduos classificados clinicamente como livres de cárie, após o exame radiográfico apresentaram lesões cariosas com profundidade em dentina (HOPCRAFT;MORGAN, 2005), confirmando o importante papel do exame radiográfico no diagnóstico de cárie da superfície proximal.

Na tentativa de relacionar a lesão de cárie proximal em dentes decíduos e permanentes, Méjare et al. (2001) avaliaram, em um estudo retrospectivo, o padrão de cárie da superfície mesial do primeiro molar permanente relacionado com a condição da distal do segundo molar decíduo em 374 crianças de seis a 12 anos de idade. $O$ índice de cárie no primeiro molar permanente foi 15 vezes maior quando o segundo molar decíduo apresentava lesão de cárie na superfície distal. A extensão radiográfica da lesão no segundo molar decíduo não representou diferença significativa na incidência de cárie no primeiro molar permanente. A presença de le-

* Mestre em Odontologia - Programa de Pós-Graduação em Odontologia, Clínica Odontológica - Odontopediatria ** Doutora em Estomatologia USP; Professora da Disciplina de Histologia da Faculdade de Odontologia da UFRGS *** Doutor em Odontopediatria USP; Professor da Disciplina de Odontopediatria da Faculdade de Odontologia da UFRGS 

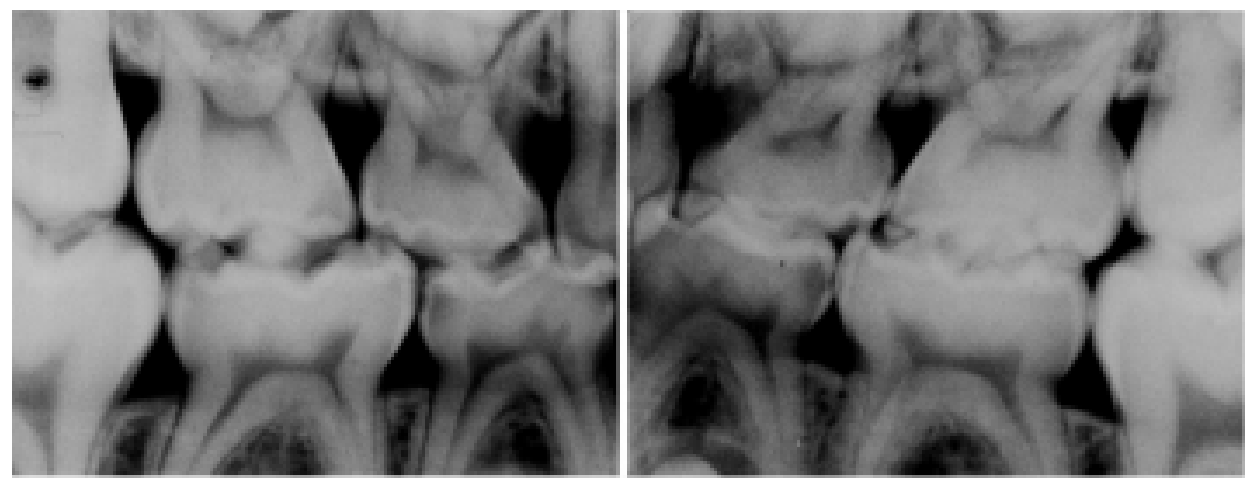

Figura 1 - Lesões proximais em superfícies homólogas do mesmo paciente (84D/74D).

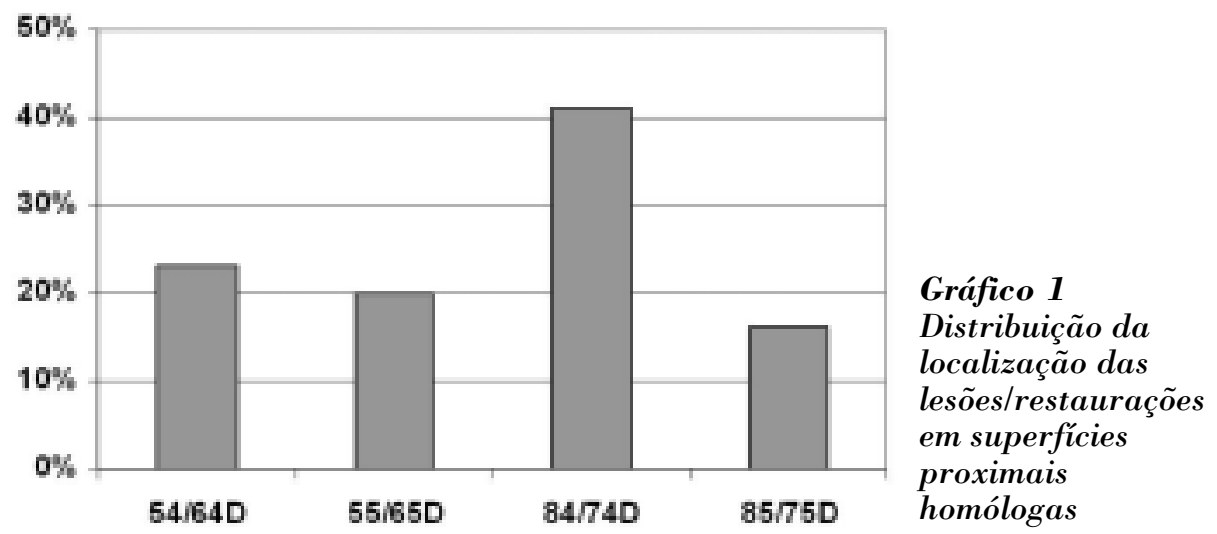

são de cárie dentinária e restauração entre o primeiro e o segundo molares decíduos aumenta em aproximadamente três vezes a chance do indivíduo desenvolver cárie entre o segundo molar decíduo e o primeiro molar permanente. Outro estudo com adultos jovens mostrou que a média de superfícies com lesões em esmalte é de aproximadamente três por indivíduo e ainda, que $20 \%$ da amostra apresentou pelo menos quatro lesões em esmalte exclusivamente detectadas pela radiografia (POORTERMAN et al., 2002).

A luz destas considerações, o objetivo deste estudo transversal observacional foi avaliar a experiência de cárie na superfície proximal de molares decíduos quanto à ocorrência de comportamento bilateral e ao envolvimento de superfícies adjacentes. Desta forma, tem-se a intenção de propiciar ao cirurgião-dentista subsídios para a indicação do exame radiográfico para o diagnóstico de cárie proximal em molares decíduos.

\section{MATERIAIS E MÉTODOS}

\section{Amostra}

A amostra foi selecionada do arquivo de documentação de pacientes do Ambulatório de Odontopediatria da Universidade Federal do Rio Grande do Sul. Tal ambulatório é responsável pelo atendimento clínico de crianças de 0 a 12 anos nóstico de cárie. sença de mais de um par de radiografias do paciente, apenas o de data mais recente foi incluído na análise.

Após a seleção inicial, o examinador avaliou as radiografias aleatoriamente (codificadas para que o mesmo não identificasse os pares correspondentes a cada indivíduo) e classificou as superfícies proximais de acordo com os seguintes critérios:

- Hígida: ausência de radiolucidez na superfície proximal de molares decíduos;

- Lesão de cárie e/ou restauração proximal: presença de radiolucidez compatível com lesão cariosa e/ou restauração na superfície proximal de molares decíduos.

Examinador e calibragem

Um examinador foi calibrado quanto à interpretação radiográfica através do exame duplicado de um conjunto de 50 radiografias interproximais infantis com diferentes manifestações de lesões de cárie proximal, alcançando um Kappa = 0,84 . Os critérios para análise radiográfica foram: ausência de radiolucidez, radiolucidez em esmalte, radiolucidez na metade externa da dentina, radiolucidez na metade interna da dentina e presença de restauração envolvendo a face proximal.

\section{Análise dos dados}

Os resultados foram apresentados de que buscam atendimento Odontológico na Instituição. Do total de 670 fichas arquivadas, 208 possuíam radiografias interproximais bilaterais.

\section{Critérios de Seleção}

$\mathrm{O}$ examinador avaliou as radiografias e selecionou pares bilaterais de acordo com os seguintes critérios:

- Inclusão: radiografias interproximais apresentando radiolucidez compatível com lesão cariosa ou restauração proximal em pelo menos um molar decíduo, sem sobreposição das superfícies proximais e com boa nitidez e contraste para o diag-

- Exclusão: radiografias com má qualidade da imagem (artefatos de técnica, contraste e nitidez inadequados e sobreposição das superfícies proximais de molares decíduos), ausência de molares decíduos, ausência de lesões cariosas, presença de destruição coronária e/ou restaurações coronárias amplas que inviabilizassem o diagnóstico de lesão proximal.

\section{Avaliação das radiografias}

$O$ exame das radiografias foi realizado a olho descoberto, em sala com mínima luminosidade, utilizando negatoscópio e moldura preta.

Um total de 64 fichas perfizeram os critérios previamente descritos. Na pre- forma descritiva, através do percentual de lesões/restaurações presentes nas superfícies homólogas, superfícies adjacentes e dos em gráficos de freqüências.

\section{RESULTADOS}

Dos 64 pacientes (pares de radiografias) incluídos no estudo, 81,25\% apresentaram radiolucidez compatível com lesões de cárie/restaurações proximais em superfícies homólogas (figura 1). Dentre esses, $58 \%$ apresentaram mais de um par de superfícies homólogas com radiolucidez.

Em $48 \%$ das crianças, sempre que uma superfície proximal apresentava radiolucidez, o mesmo ocorria com a face homóloga. Ou seja, se o paciente apresentasse duas lesões proximais no lado direito da arcada, as duas superfícies correspondentes dos dentes homólogos também apresentavam lesão ou restauração proximal diagnosticadas ao exame radiográfico.

O comportamento bilateral de lesões cariosas proximais foi mais freqüentemente observado na arcada inferior (58\%), As superfícies distais dos primeiros molares inferiores $(74 / 84)$ foram as mais observadas quanto à ocorrência bilateral (gráfico 1). em apenas uma das superfícies e ilustra- 
A presença de lesões cariosas acometendo ambas superfícies de contato (distal do primeiro molar e mesial do segundo, figura 2), foi observada em $59 \%$ das radiografias examinadas, sendo a sua distribuição semelhante em todos os sítios (gráfico 2).

\section{DISCUSSÃO}

Este estudo mostra que dentre crianças com lesões de cárie proximais identificadas radiograficamente, uma grande proporção $(81,25 \%)$ apresentou lesões em superfícies homólogas. Apesar de não se observar na literatura este tipo de avaliação, alguns estudos já relataram ser freqüente a ocorrência de mais de uma lesão proximal na cavidade bucal, independentemente da faixa etária do indivíduo (MEJARE et al., 2001; POORTERMAN et al., 2002). Poorterman et al. (2002), em um estudo com pacientes adolescentes e adultos jovens, observaram um média de três superfícies com lesões de cárie em esmalte, adiciona-se ainda o fato de que $20 \%$ desses indivíduos apresentaram quatro ou mais lesões em esmalte exclusivamente detectadas pela radiografia.

Outro dado importante registrado neste estudo foi a proporção de $58 \%$ da amostra com lesões em superfícies homólogas que apresentaram mais de um par dentário nestas condições. Isto significa que não apenas a presença de mais de uma lesão proximal na cavidade bucal seja um achado freqüente, mas também o acometimento de mais de um par de superfícies homólogas. Essa situação nos remete à importância do exame radiográfico na investigação de lesões proximais em todos os sítios da cavidade bucal, especialmente porque a literatura relata grande número de lesões proximais não detectadas ao exame clínico (Anderson et al., 2005).

Estudos tranversais não são capazes de identificar a temporalidade dos fatos ocorridos (KLEIN;BLOCH, 2002), portanto, a presente avaliação realizada com uma amostra de conveniencia de indivíduos de um centro de referência tem restritas inferências populacionais. Entretanto, estudos longitudinais mostram o risco aumentado de desenvolvimento de novas lesões cariosas em períodos específicos da vida do indivíduo, principalmente quando lesões cariosas proximais já haviam sido diagnosticadas na fase de dentição mista (MEJARE et al., 2004). Isso demonstra que o desequilíbrio do processo des-remineralização não é um evento isolado em um sítio, mas acomete a cavidade bucal como um todo (FEJER-
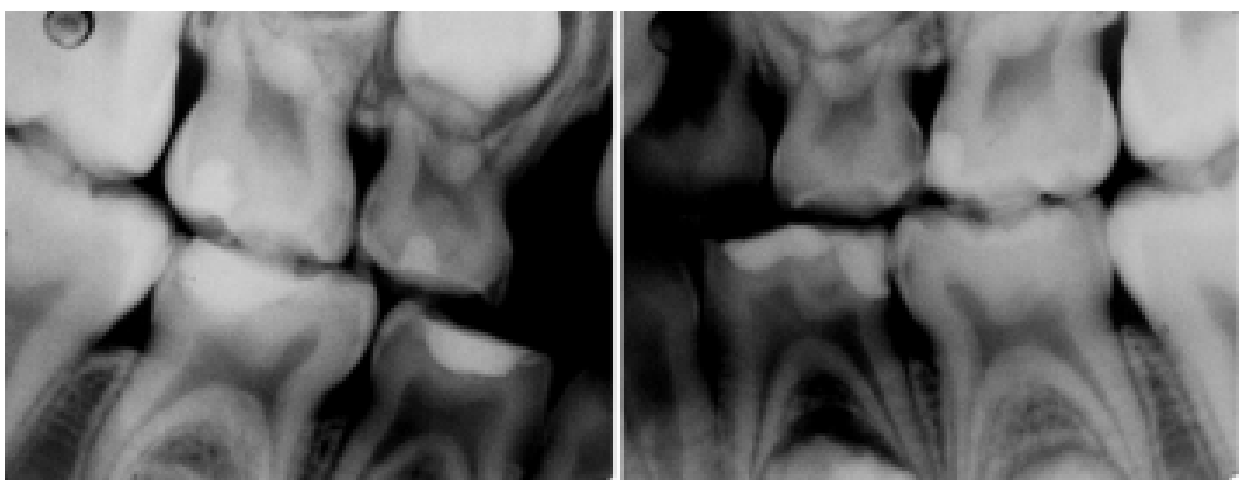

Figura 2 - Lesões/restaurações proximais em superfícies adjacentes (55M/54D; 85M/ $84 D ; 64 D / 65 M)$.

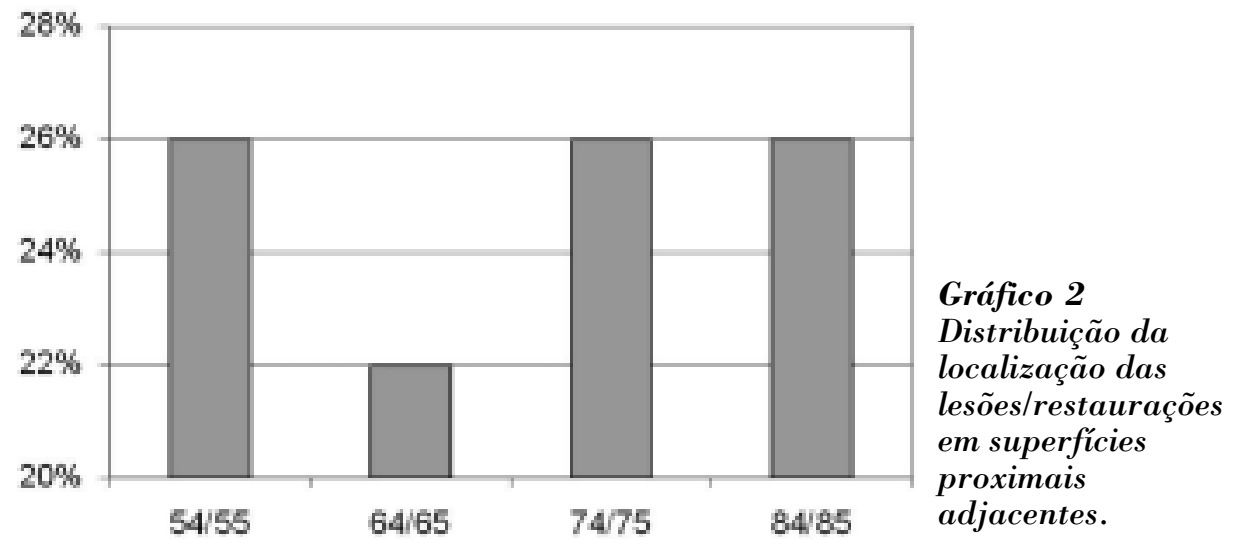

SKOV, 2004), sendo plausível o diagnóstico de lesões de cárie em mais de uma superfície dentária proximal da cavidade bucal.

Em relação a amostra deste estudo, as radiografias foram coletadas no arquivo de fichas do Ambulatório de Odontopediatria da UFRGS. Sendo este um centro de referência local para atendimento público, apresenta particularidades na expressão da doença cárie que não pode ser extrapolada a toda a população. Espera-se, portanto, que a severidade, prevalência e, conseqüientemente, a freqüência de lesões cariosas homólogas e adjacentes estejam superestimadas. Tal informação possa ser útil para embasar o planejamento de estudos epidemiológicos, contemplando este tipo de análise, a fim de avaliarmos na população a ocorrência deste comportamento das lesões cariosas. Cabe salientar ainda, que estudos epidemiológicos (MEJARE et al., 2001; POORTERMAN et al., 2002) já mostraram a ocorrência de lesões proximais em diversos sítios da cavidade bucal, justificando a necessidade da investigação clínica por outras lesões cariosas quando houver a detecção de lesão cariosa proximal.

Os achados do presente estudo indi- cam que, quando do diagnóstico de uma lesão cariosa proximal, é de fundamental importância investigar outros sítios proximais da cavidade bucal.

\section{CONCLUSÕES}

De acordo com a metodologia utilizada, pode-se concluir que

- lesões de cárie nas superfícies proximais de molares decíduos apresentam comportamento bilateral;

- a presença de lesões em ambas as superfícies de contato é um achado freqüiente.

\section{ABSTRACT}

The aim of this cross-sectional study was to evaluate the bilateral and adjacent occurrence of approximal caries on primary dentition. The study sample was comprised by patients' files from children attended at the Pediatric Dental Clinic of the Faculty of Dentistry at the Federal University of Rio Grande do Sul. From a total of 670 files, 208 had bilateral bitewing radiographs and were selected for the study. The children's files with any approximal caries lesion/restoration, adequate brightness and contrast degree and without approximal superposition 
were selected for the analysis (kappa 0.84). Radiographs were evaluated at random and the approximal caries lesion and restoration were recorded. The occurrence of homologous and adjacent approximal caries lesions on deciduous molars was reported. $81 \%$ of the sample (64 pairs of bitewing radiographs) presented caries lesion in homologous approximal surfaces and $58 \%$ of these individuals showed more than one pair of these lesions. The most frequently affected surface $(41 \%)$ was the distal surface of primary molars. $59 \%$ of the children presented caries lesion/restoration on both adjacent approximal surfaces.

Considering the methodology of the present study, it can be concluded that caries lesions in deciduous molars present a bilateral pattern of occurrence in homologous and adjacent approximal surfaces. These findings must be considered during clinical investigation of caries lesions in children with past caries experience.

\section{KEYWORDS}

Dental caries. Deciduous teeth. Approximal caries lesion. Bitewing radiograph.

\section{REFERÊNCIAS}

ANDERSON, M., et al. Detection of Approximal Caries in 5-Year-Old Swedish Children. Caries Res., Basel, v.39, no.2, p.92-99, Mar./Apr., 2005.

FEJERSKOV, O. Changing Paradigms in Concepts on Dental Caries: Consequences for Oral Health Care. Cari- es Res., Basel, v.38, no.3, p.182-191, May/Jun, 2004.

GRONDAHL, H. G., et al. Approximal Caries and Frequency of Bitewing Examinations in Swedish Children and Adolescents. Community Dent. Oral Epidemiol., Copenhagen, v.20, no.1, p.20-24, Feb, 1992.

HOPCRAFT, M. S.; MORGAN, M. V. Comparison of Radiographic and Clinical Diagnosis of Approximal and Occlusal Dental Caries in a Young Adult Population. Community Dent. Oral Epidemiol., Copenhagen, v.33, no.3, p.212-218, June, 2005.

KLEIN, C. H.; BLOCH, K. V. Estudos Seccionais. In: Medronho, R. A. Epidemiologia. São Paulo: Estudos Seccionais, 2002. Cap. 9, p. 125-150

MACHIULSKIENE, V., et al. A Comparison of Clinical and Radiographic Caries Diagnoses in Posterior Teeth of 12-Year-Old Lithuanian Children. Caries Res., v.33, no.5, p.340-348, Sept./Oct., 1999.

MEJARE, I., et al. Influence of Approximal Caries in Primary Molars on Caries Rate for the Mesial Surface of the First Permanent Molar in Swedish Children from 6 to 12 Years of Age. Caries Res., v.35, no.3, p.178-185, May/June, 2001.

Caries Incidence and Lesion Progression from Adolescence to Young Adulthood: A Prospective 15-
Year Cohort Study in Sweden. Caries Res., v.38, no.2, p.130-141, Mar/ Apr., 2004.

PITTS, N. B. The Bitewing Examination as a Preventive Aid to the Control of Approximal Caries. Clin. Prev. Dent., v.6, no.1, p.12-15, Jan./Feb., 1984.

POORTERMAN, J. H., et al. Radiographic Prevalence of Approximal Enamel Lesions and Relationship with Dentine Lesions and Restorations in Dutch Adolescents. Int. Dent. J., v.52, no.1, p.15-19, Feb, 2002.

UPRICHARD, K. K., et al. Comparison of Direct Digital and Conventional Radiography for the Detection of Proximal Surface Caries in the Mixed Dentition. Pediatr. Dent., v.22, no.1, p.9-15, Jan./Feb., 2000.

VAN RIJKOM, H. M.; VERDONSCHOT, E. H. Factors Involved in Validity Measurements of Diagnostic Tests for Approximal Caries-a Meta-Analysis. Caries Res., v.29, no.5, p.364-370, 1995.

WENZEL, A. Digital Radiography and Caries Diagnosis. Dentomaxillofac. Radiol., v.27, no.1, p.3-11, Jan, 1998.

Endereço para correspondência: Faculdade de Odontologia da UFRGS Rua Ramiro Barcelos, 2492 CEP 90035-003 - Porto Alegre - RS 